\title{
Current methods for the assessment of skin microcirculation: Part 1
}

\author{
Jolanta Neubauer-Geryk ${ }^{1}$, Magdalena Hoffmann², Melanie Wielicka ${ }^{3}$, Katarzyna Piec $^{4}$, Grzegorz Kozera $^{4}$, \\ Maciej Brzeziński ${ }^{5}$, Leszek Bieniaszewski ${ }^{1}$
}

${ }^{1}$ Clinical Physiology Unit, Medical Simulation Centre, Medical University of Gdansk, Gdansk, Poland

${ }^{2}$ Medical Centre, Pruszcz Gdanski, Poland

${ }^{3}$ Clinical Physiology Unit, Medical Simulation Centre/Students' Scientific Circle, Medical University of Gdansk, Gdansk, Poland ${ }^{4}$ Department of Neurology, Collegium Medicum in Bydgoszcz, Nicolaus Copernicus University in Torun, Poland

${ }^{5}$ Department of Cardiac and Vascular Surgery, Medical University of Gdansk, Gdansk, Poland

Adv Dermatol Allergol 2019; XXXVI (3): 247-254

DOI: https://doi.org/10.5114/ada.2019.83656

\begin{abstract}
Microcirculation accounts for about $99 \%$ of blood vessels in adults and mediates between the arterial and venous parts of the cardiovascular system, both structurally and functionally. Skin microcirculation consists of two vascular plexuses: superficial and deep. Microcirculation includes vessels with a diameter of less than $150 \mu \mathrm{m}$, i.e. arteries, small veins, lymphatic vessels and arteriovenous anastomoses, which build the microcirculation unit. Skin microcirculation may be affected both in systemic pathologies and specific skin disorders. Several non-invasive techniques are available to assess the skin microcirculation. The clinical value is recognised for capillaroscopy and videocapillaroscopy, laser Doppler flowmetry thermography and transcutaneous oxygen measurement. The list of methods that may be used in clinical research also includes: photoplethysmography, orthogonal spectral polarization, near infrared spectroscopy and tissue reflectance spectrophotometry and optical coherence tomography.
\end{abstract}

Key words: skin microcirculation, methods.

\section{Introduction}

Microcirculation accounts for about $99 \%$ of blood vessels in adults and mediates between the arterial and venous parts of the cardiovascular system, both structurally and functionally. It includes vessels with a diameter of less than $150 \mu \mathrm{m}$, i.e. arteries, small veins, lymphatic vessels and arteriovenous anastomoses, which build the microcirculation unit. According to the definition based on both, the diameter of the vessel and the vascular muscle response to the pressure difference, all vessels that constrict due to elevated blood pressure contribute to the microcirculatory unit (Figure 1). Microcirculation is used to exchange nutrients and metabolites between blood and tissue, protect against significant fluctuations in hydrostatic pressure in the capillary network and decrease peripheral vascular resistance.

Skin microcirculation consists of two vascular plexuses: superficial at a depth of 400-500 $\mu \mathrm{m}$ and deep located at a depth of $1.9 \mathrm{~mm}$ below the skin surface. From the upper layer to the basal one, arterial capillaries with cross-section of $10 \mu \mathrm{m}$ loop away. Ascending arterioles and descending venules are paired as they connect the two plexuses by arteriovenous anastomoses (Figure 2).

Skin microcirculation may be affected both in systemic pathologies and specific skin disorders. Septic and allergic shocks, global hypoxia, hypoxia-reperfusion injuries, coagulation disorders and activation of the immune system affect the skin microcirculation function. The microvascular skin impairment is pathognomonic for systemic disorders including connective tissue diseases, vasculitis, Raynaud's phenomenon, diabetes mellitus, chronic kidney disease and arterial hypertension. The microcirculation function may be also influenced by temperature, systemic blood pressure, general body condition and nutrition status, stress, use of nicotine, mental and physical activity and aging.

Some of the physiological factors that influence microvessels may be used as provocative tests. The most common are change in the body position (to assess venoarterial reflex with vasoconstriction), heating and cooling

Address for correspondence: Jolanta Neubauer-Geryk MD, PhD, Clinical Physiology Unit, Medical Simulation Centre, Medical University of Gdansk, 25 Dębowa St, 80-204 Gdansk, Poland, phone: +48 58349 11 89, e-mail: jolaneub@gumed.edu.pl Received: 19.09.2017, accepted: 22.02.2018. 


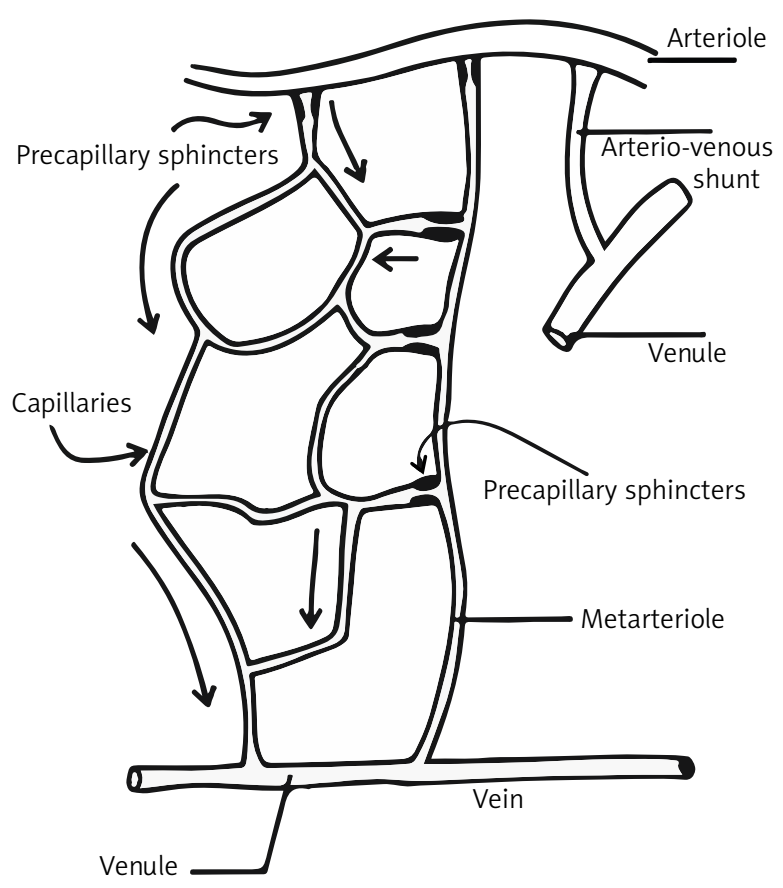

Figure 1. Microcirculation unit

(to measure the range of vasoconstriction and vasodilatation e.g. Raynaud's phenomenon), compression (to determine the post-occlusive hyperaemia, PORH) and stimulation of $C$ fibers.

Currently, several non-invasive techniques are available to assess the skin microcirculation. A clinical value is recognised for: capillaroscopy and videocapillaroscopy, laser Doppler flowmetry, thermography and transcutaneous oxygen measurement $\left(\mathrm{tcpO}_{2}\right)$. The list of methods that may be used in clinical research also includes: photoplethysmography, orthogonal spectral polarization, near infrared spectroscopy (NIRS) and tissue reflectance spectrophotometry and optical coherence tomography (OCT) (Table 1).

\section{Capillaroscopy}

Capillaroscopy is based on the non-invasive in-vivo assessment of blood microcirculation within the skin and mucous membranes. It involves the viewing of capillary loops of nailfolds, usually in the fingers (walls of the nails in fingers $I I-V$ or $I I-V)$, rarely in the toes. Because capillaries in the nailfolds are arranged parallel to the surface of the skin, it is possible to accurately evaluate them. In other skin areas, capillaries are perpendicular to the surface of the skin and, therefore, only peaks of the capillary loops are visible in the test.

Capillaroscopy allows the repeatable evaluation of the structure and morphology of the selected microcirculation fragment (Figure 3). Capillaroscopy assesses

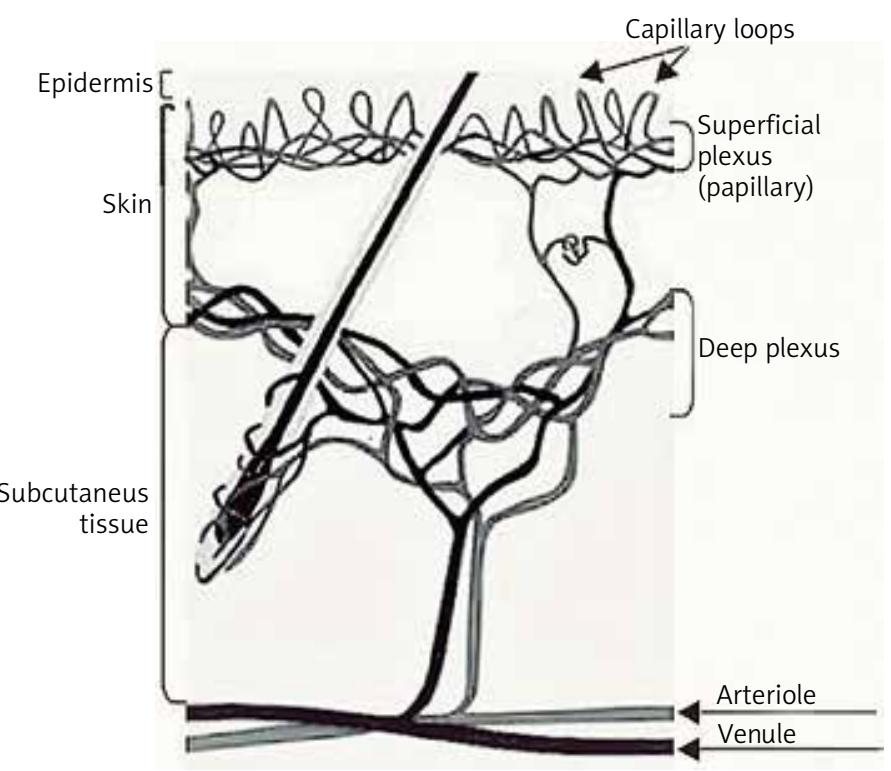

Figure 2. Skin microcirculation

the nail plate, skin clarity, capillary number, their size, shape and location, the blood flow through the loops, the presence of petechiae, capillary deformity and sub papillary venous plexuses (Table 2) [1-4]. In traditional capillaroscopy, an optical microscope with lateral illumination is used, which allows a magnification of 20-100 times petechiae (Figure 3). Fluorescence capillaroscopy and videocapillaroscopy are also in use.

In a healthy subject, the picture of the wall of the nail of the same finger does not change significantly over time. Thus, the presence of lesions revealed by a capillaroscopic examination may confirm a specific pathology of microcirculation. Usually, giant capillaries, megacapillaries, broad loops, tortuous capillaries and the areas of a reduced number and heterogeneous arrangement of capillaries are observed. The result of capillaroscopy is often an important clue to making the final diagnosis and a valuable complement to serological tests and a clinical picture. However, capillaroscopy requires a lot of experience when assessing the image. In addition, we should take into account the effects of medications like $\beta$-blockers and calcium channel blockers, which may cause changes in the evaluated vessels [1].

Before the test, it is necessary to avoid cosmetic procedures on the fingers and toes for about 3 weeks. Because the diameter of the capillaries depends on external factors, such as temperature, stress or stimulants, the test should be performed in a quiet room, at a stable temperature of $>20^{\circ} \mathrm{C}$, providing heat adaptation time of about 15-20 min and mental comfort in a sitting position. Drinking coffee, tea and smoking cigarettes should be avoided before the test. Prior to capillaroscopy, the selected area of nailfold is cleaned and immersion oil is 
applied to improve visibility of the capillaries and to increase the translucency of the stratum corneum [1-4].

The skin capillaries are examined by a stereomicroscope with spot-lighting. Images are taken and stored using a digital camera attached to the capillaroscope. The image analysis allows to determine the mean distance between successive capillaries (DISTANCE) and the ratio between the cross sectional area of the capillaries and the total area of determined rows (COVERAGE). For the examination of skin microcirculation reactivity, capillaroscopy is performed twice, before and after a stimulus, such as a 30-minute $\mathrm{L}$-arginine infusion. The skin microcirculation reactivity is then expressed as the difference between the values of distance ( $\triangle$ DISTANCE) and coverage ( $\triangle$ COVERAGE) obtained before and after the test.

The quantitative method for evaluating cutaneous microcirculation in the nailfold is done offline by determining the coverage and the average distance between capillaries in the analysed area with dedicated software (Figures 4, 5) [5].

Capillaroscopy is typically used in the diagnosis of rheumatic diseases, including inflammatory arthritis, systemic lupus erythematosus and the primary and secondary form of Raynaud's phenomenon, which is the main indication for capillaroscopy [2-4]. The test has also been used in skin diseases, such as rosacea [4]. It is also helpful in evaluating tuberculous lesions and monitoring outcomes of treatments, for instance in psoriasis. Capillaroscopy is applied in vascular surgery to assess capillary flow dysfunction in patients with diabetic microangiopathy $[5,6]$, chronic venous insufficiency and lower limb ischaemia [7]. The examination is also used in the diagnostics of arterial hypertension, neurological disorders and frostbite evaluation [8].

\section{Videocapillaroscopy}

Videocapillaroscopy, when compared to the traditional capillaroscopy, provides better magnifying power, storing pictures and off-line analysis $[9,10]$. The procedure of preparing a patient for a videocapillaroscopy is similar to traditional capillaroscopy. Videocapillaroscopy assessment might be performed in many points of the human body. Nonetheless, the nailfold, owing to the characteristic layout of its capillary vessels, tends to be the most commonly examined area. Similarly to traditional capillaroscopy, videocapillaroscopy

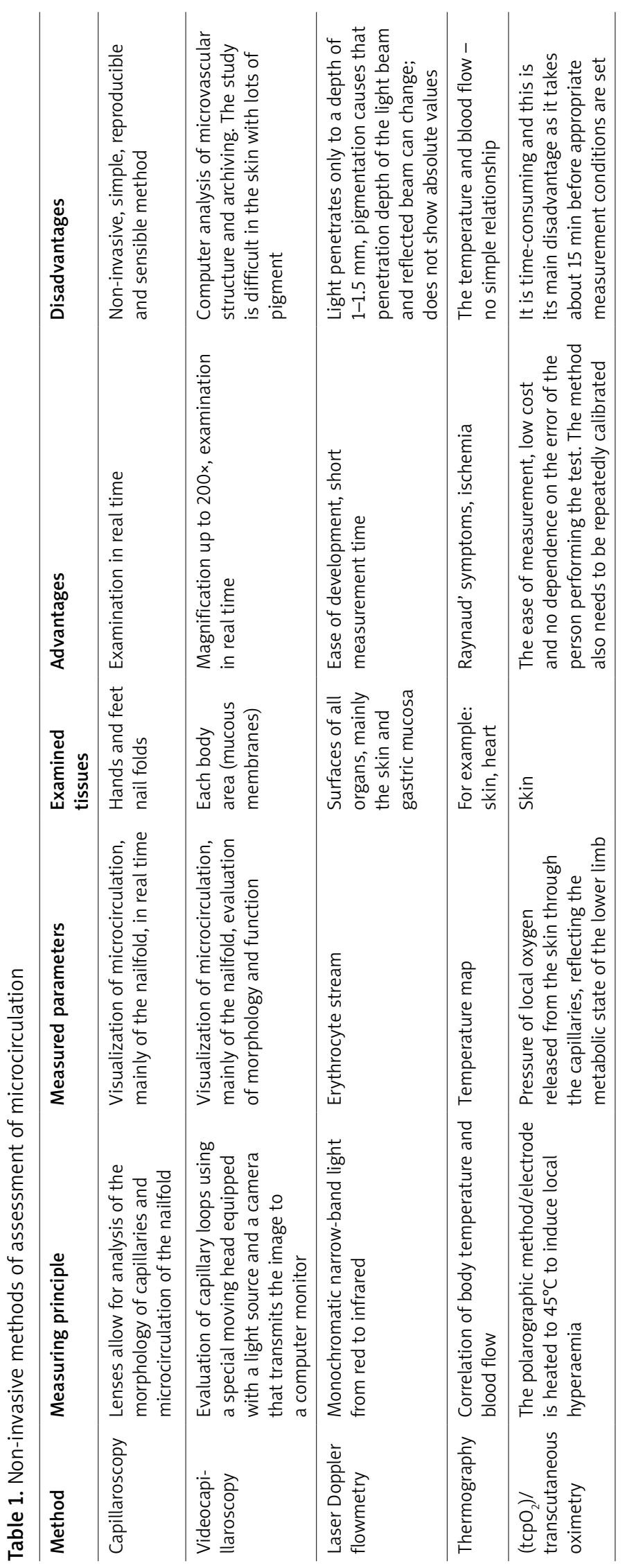




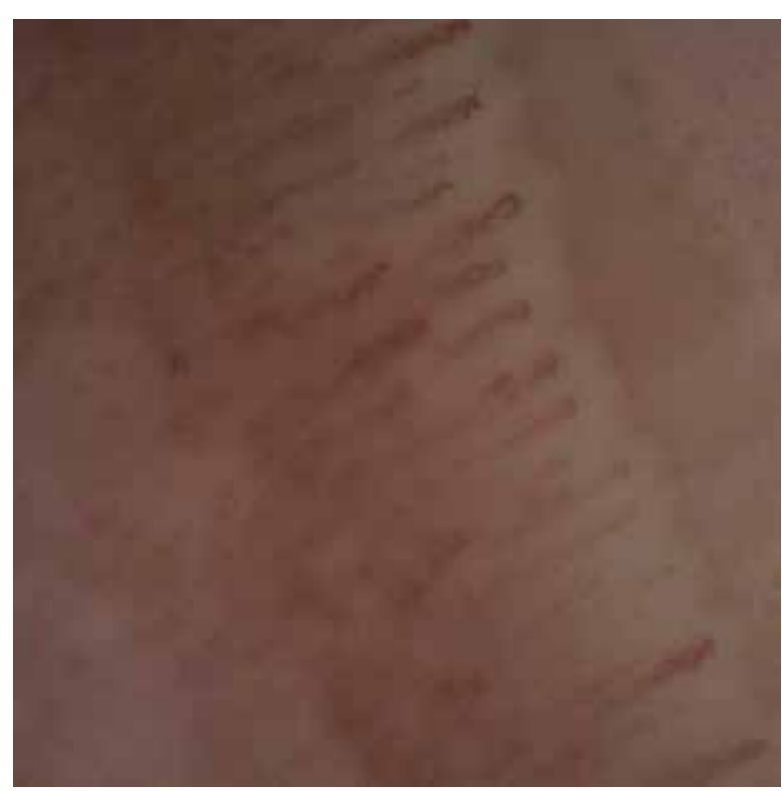

Figure 3. Image from the capillaroscopy (from the Clinical Physiology Unit, Medical Simulation Centre, Medical University of Gdansk, Gdansk, Poland)

enables examination of the dynamics of changes in microcirculation both in rheumatologic and non-rheumatologic diseases, primarily dermatologic, neurologic as well as civilization diseases, such as hypertension and diabetes. It may be also applied in the assessment of microangiopathy in patients with systemic autoimmune diseases (including rheumatoid arthritis and juvenile idiopathic arthritis), threatened by premature development of sclerosis. Thus, it can be used to identify young patients with a high risk of cardiovascular disease.

\section{Laser Doppler flowmetry and laser Doppler imaging}

Laser Doppler flowmetry (LDF) is a method that allows for non-invasive real-time recording of microcirculation [11]. The examination can be performed on the surface of each organ; most often it is carried out on the skin or mucous membrane of the stomach or mouth. In recent years, many publications have been devoted to this research method, due to its easy-to-use, short measurement times and easy training of the person performing the test. The biggest disadvantage of LDF is its sensitivity to unstable test conditions [12]. In addition, only a small volume of tissue can be registered and flow values are not obtained in absolute units.

LDF uses a $2 \mathrm{~mW}$ helium-neon laser with the monochromatic laser beam in the narrow infrared band light that penetrates and spreads within the examined tissue to a depth of about $1 \mathrm{~mm}$. In the tested tissue, some of the laser rays are absorbed, some of them are reflected from the immobile matter (protein, keratin, melanin, haemoglobin) without changing their frequency, while the rest bounces off the moving red blood cells with altered frequency due to the Doppler effect $[1,13]$. The intensity of the signal is directly proportional to the number and speed of red blood cells in the examined area. Returning light is then analysed using a photodetection system, which generates voltage directly proportional to the speed and number of moving cells in the blood of the tested area $[14,15]$.

The main limitation of laser-counter technology is the fact that it is not possible to measure absolute perfusion values (in $\mathrm{ml} / \mathrm{min}$ ). The device registers the blood supply in the tested area of the tissue in the perfusion unit (PU) or in millivolts $(1 \mathrm{PU}=10 \mathrm{mV})$. Most authors

Table 2. The microcirculation properties assessed by capillaroscopic examination

\begin{tabular}{|c|c|}
\hline Capillaroscopic parameter & Normal image \\
\hline Skin transparency and visibility & Transparent, capillaries clearly visible \\
\hline Pericapillary oedema & Absent \\
\hline Subpapillary venous plexus & Visible in up to $30 \%$ of healthy people \\
\hline Capillary array and architecture & Straight capillaries, perpendicular to the nailfold \\
\hline Capillary morphology & U-shaped \\
\hline Capillary loop diameter & $<20 \mu \mathrm{m}$ \\
\hline Tortuosity & Usually absent \\
\hline Dilated $(20-50 \mu \mathrm{m})$ and giant $(>50 \mu \mathrm{m})$ loops & Absent \\
\hline Ramified capillaries & Absent \\
\hline Haemorrhages, hemosiderin deposits & Usually absent, may be present after local trauma \\
\hline Capillary density & 9-13 in 1 linear millimetre \\
\hline Neoangiogenesis & Absent \\
\hline Avascular areas (distance between 2 loops > $500 \mu \mathrm{m}$ ) & Absent \\
\hline Capillary blood flow & Dynamic, no stasis or thrombosis \\
\hline
\end{tabular}




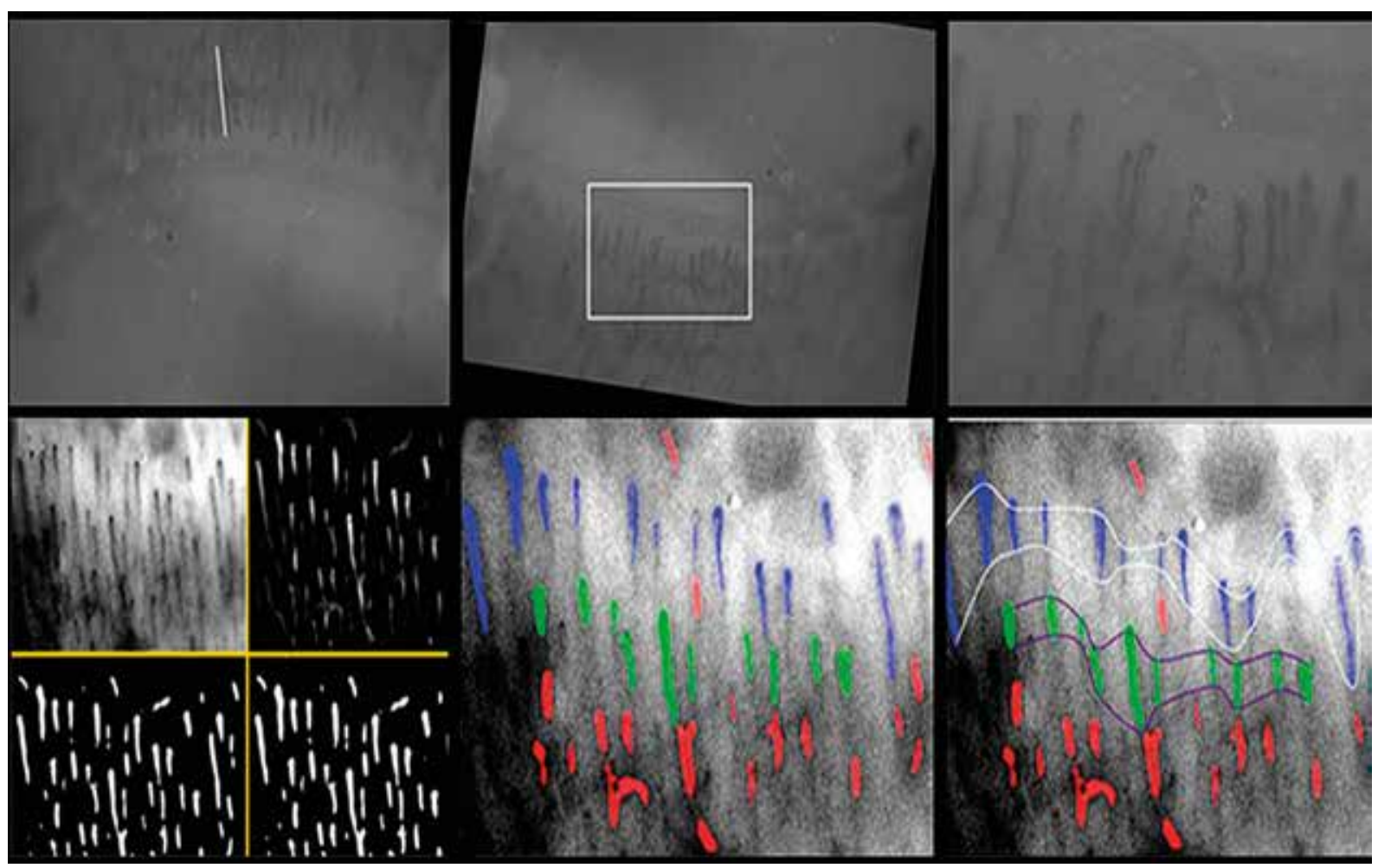

Figure 4. Illustration of the program for the detection of capillaries - steps (from the Clinical Physiology Unit, Medical Simulation Centre, Medical University of Gdansk, Gdansk, Poland)
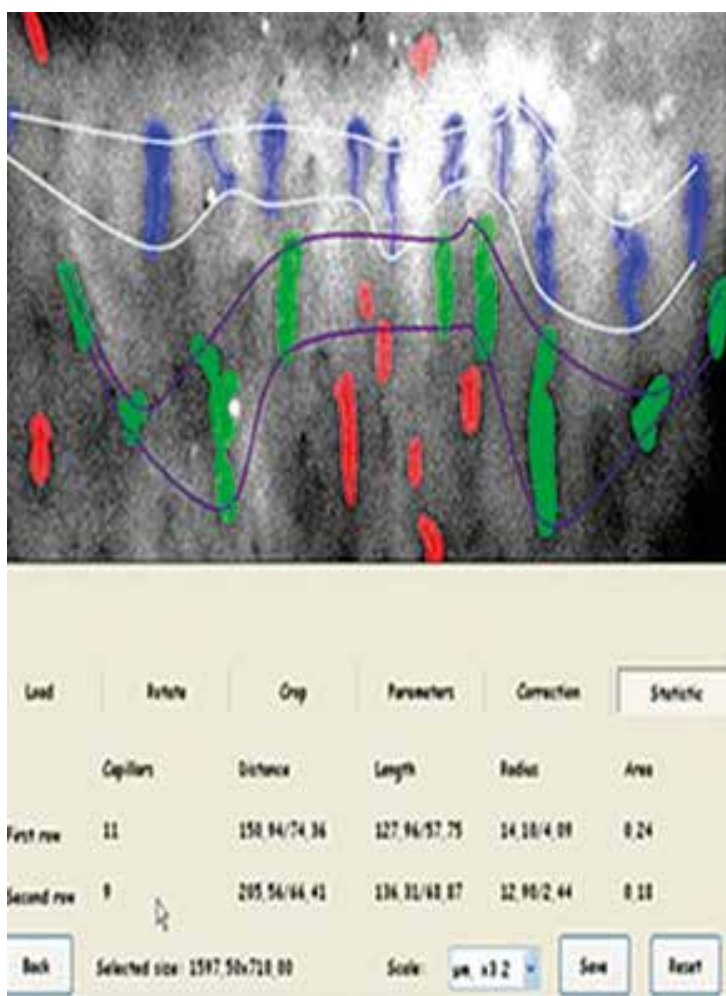
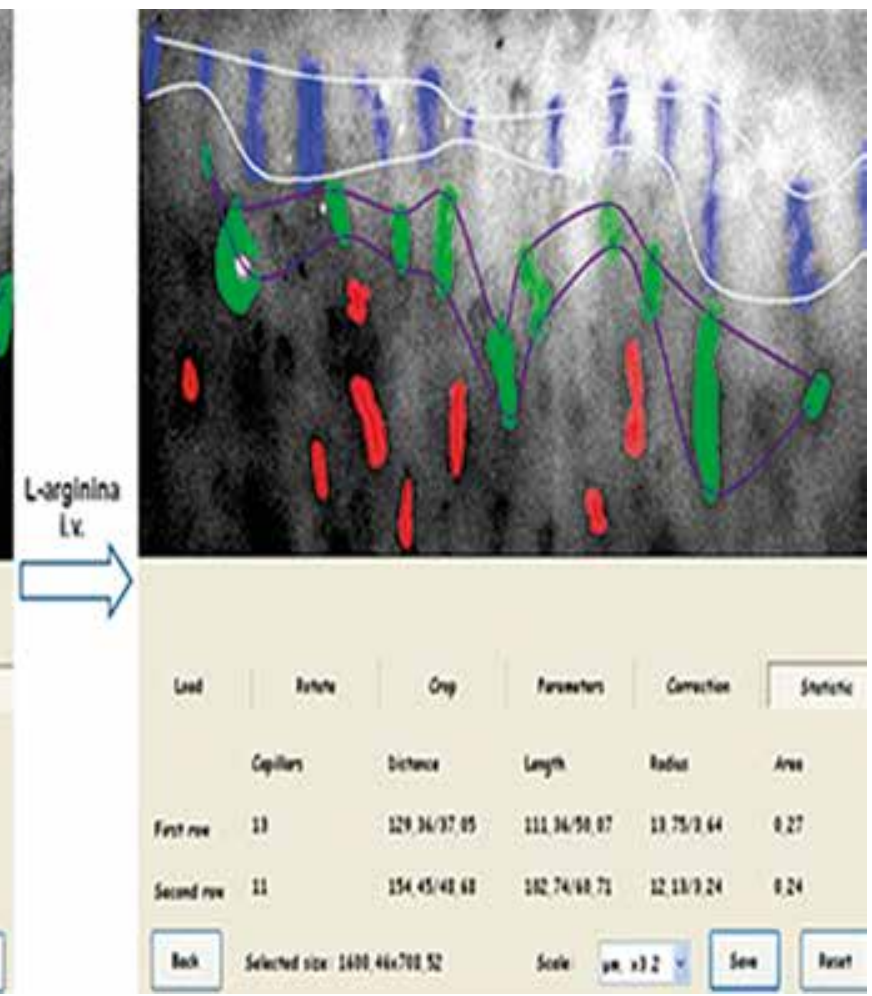

Figure 5. Illustration of the program for the detection of capillaries - calculations (from the Clinical Physiology Unit, Medical Simulation Centre, Medical University of Gdansk, Gdansk, Poland) 

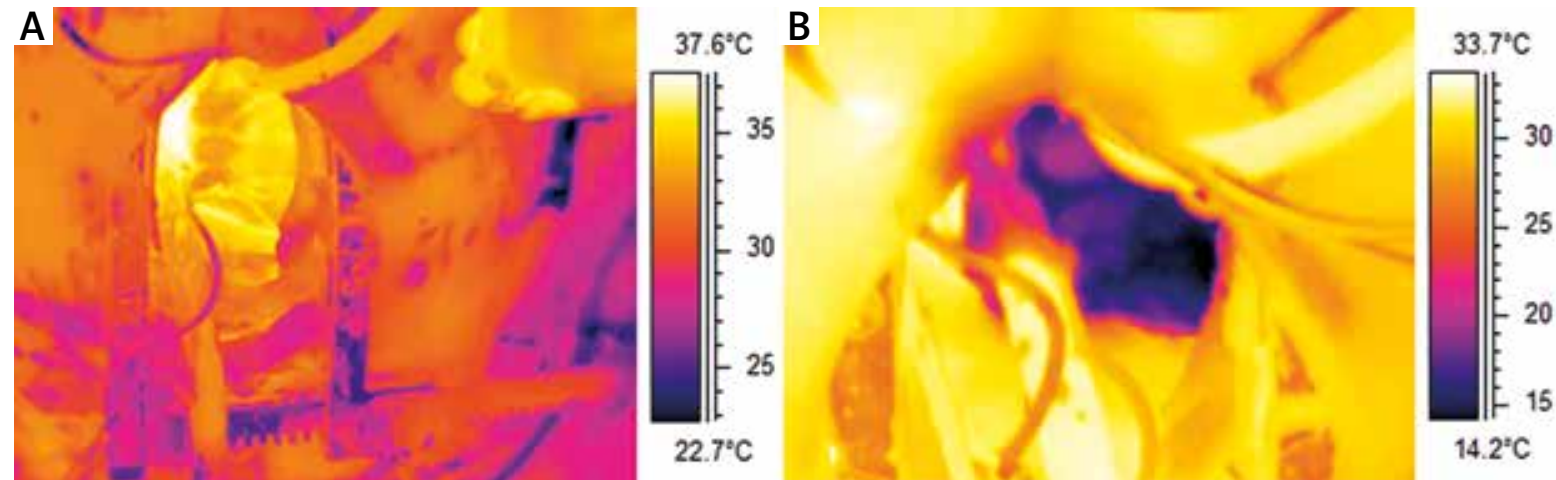

Figure 6. The heart before (A) and after cardioplegia (B). The entire sequence was recorded at $30 \mathrm{FPS}$ as a radiometric file by an infrared camera Flir A325 with 320x240px detector with a temperature resolution of $0.05^{\circ} \mathrm{C}$. With the permission of M. Brzeziński, Department of Cardiac and Vascular Surgery, Medical University of Gdansk, Poland; G. Strugała, Gdansk University of Technology, Faculty of Mechanical Engineering, Gdansk, Poland

present results as cutaneous vascular conductance (CVC), dividing blood flow by mean blood pressure ( $\mathrm{mV} /$ $\mathrm{mm} \mathrm{Hg).} \mathrm{This} \mathrm{approach} \mathrm{seems} \mathrm{appropriate} \mathrm{because} \mathrm{it}$ takes into account possible differences and changes in blood pressure [14]. The analysis is also sensitive to changes in testing conditions. These problems can be eliminated by performing provocative tests such as reactive congestion caused by skin heating, controlled ischemia or iontophoresis of vasoactive substances. Thus, Roustit et al. have confirmed repeatability of the method by evaluating perfusion using single-point laser Doppler flowmetry on the finger pad after the use of post-occlusive reactive hyperaemia and local thermal hyperaemia [15].

Laser Doppler flowmetry, which is used to assess microcirculation in the skin, has shown clinical usefulness primarily in rheumatology, dermatology and surgery [16]. The LDF examination is helpful in assessing the severity of burns and facilitates the decision to initiate therapy. This method is also used to evaluate wound healing, including postoperative wounds in prosthetic surgery [17]. Laser Doppler flowmetry has also been used in the diagnosis of rheumatological diseases, such as Raynaud's phenomenon and systemic sclerosis [18]. In rheumatoid arthritis, the results of LDF correlate with the strength of pain and the severity of synovitis confirmed by ultrasound. The test may also be applied to assess the severity of psoriasis and its response to treatment. LDF is also used to evaluate microcirculation in patients with type 1 and type 2 diabetes. This test is particularly important in this group of patients because changes in the microcirculation usually occur before microangiopathic lesions [15]. LDF is also used for assessment of microcirculation in patients with sepsis, hypertension and chronic venous insufficiency.

\section{Thermography}

Thermography enables the recording of temperature, and thus creating a visualization of the heat flow in skin microcirculation. Abnormal body temperature is a natural indicator of the disease [19]. Thermography is another non-invasive test that does not require a lot of technical experience and is relatively easy to use. In addition, it is a touch-free method and the subject is not exposed to any harmful radiation or other procedures. The main disadvantages of thermography include the possibility of flow measurement only in superficial layers and high cost of equipment, which limits its availability.

The thermographic examination is based on the recording of the human body infrared radiation. Digital images of skin infrared radiation (thermograms) provide information on the distribution of temperature on the skin surface and allow to create a two-dimensional map of the skin temperature. However, thermography only enables an indirect assessment of the skin microcirculatory flow.

Thermography is used in both pre-clinical and clinical practice. It serves as a diagnostic and prognostic tool in patients with diabetes mellitus. Sivanandam et al. have demonstrated that measurement of skin temperature allows to establish a diagnosis of diabetes type 2 earlier than when using the standard glycated haemoglobin $\left(\mathrm{HbA}_{1 \mathrm{c}}\right)$ parameter [20]. Thermography may be also used to diagnose an early phase of diabetic neuropathy [21]. In plastic and reconstructive surgery, thermography is applied to identify the appropriate skin graft and, in the postoperative period, is an excellent method of perfusion monitoring within the graft and early detection of skin graft rejection. In cardiac surgery it allows to observe restricted areas of perfusion disturbances (Figure 6) [22]. Thermography helps to differentiate between the primary and secondary forms and to assess the severity of Raynaud's disease. It is also useful in rheumatoid arthritis, where recurrent inflammatory processes are accompanied by an increase in the temperature of the skin covering the joints. Infrared imaging allows for objective 


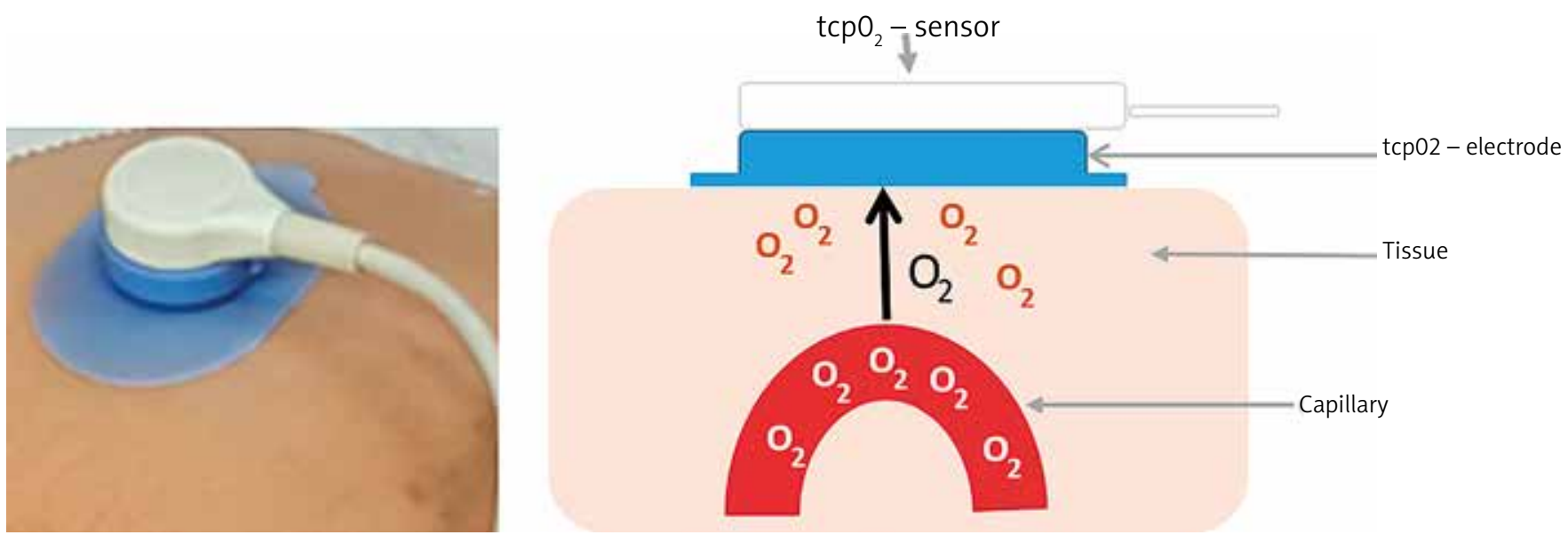

Figure 7. Transcutaneous oxygen measurement

and repetitive measurements of the intensity and extent of inflammation and for evaluation of therapy [22].

\section{Transcutaneous oxygen measurement}

Transcutaneous oxygen measurement $(\mathrm{tcpO}$ or TCOM) is a simple, non-invasive method known since 1970. It is based on the assessment of the amount of oxygen that diffuses from the capillaries through the epidermis to the electrode and thus provides information on the body's ability to deliver oxygen to the tissues (Figure 7) [23].

The TCOM is a well-documented method used for screening of vascular diseases, assessment of wound healing, evaluation of revascularization effectiveness, determining the amputation level, predicting the durability of skin grafts as well as for qualification for hyperbaric oxygen therapy [24-27]. It is relatively inexpensive compared to other methods of microcirculation evaluation. The measurement is not complicated, does not require much experience and is almost free from the examinerdependent errors. Therefore, TCOM can be carried out by a trained technician.

The TCOM measurement can be done anywhere on the skin of the entire body, usually on the dorsal surface of the foot. The test does not require prior special preparation of the patient. However, the skin must be clean and free of hair, oedema, visible large superficial vessels, skin lesions and inflammation as well as areas of increased keratosis. The main disadvantage of TCOM is that the method needs to be repeatedly calibrated, which is time-consuming and takes about 15 min before appropriate measurement [23].

$\mathrm{TcpO}_{2}$ values in the range of $50-70 \mathrm{~mm} \mathrm{Hg}$ are considered normal, those $<40 \mathrm{~mm}$ Hg suggest the possibility of wound healing disorders, $<30 \mathrm{~mm} \mathrm{Hg}$ indicate ischemia and the high probability of wound healing problems, < $20 \mathrm{~mm} \mathrm{Hg}$ announce bad prognosis with prolonged hospitalization of patients with the diabetic foot syndrome. It should be acknowledged that the $\mathrm{tcpO}_{2}$ values do not represent actual partial oxygen pressure within the wound because the electrodes are located in adjacent areas. However, experimental data suggest the relationship between the established tcpO, values and oxygen partial pressure within the wound [23, 24]. Dependence of the result on anatomical conditions of the skin and its response to a temperature increase are further disadvantages.

\section{Conflict of interest}

The authors declare no conflict of interest.

\section{References}

1. Cutolo M, Sulli A, Secchi ME. The contribution of capillaroscopy to the differential diagnosis of connective autoimmune diseases. Best Pract Res Clin Rheumatol 2007; 21: 1093-108.

2. Cutolo M. Atlas of Capillaroscopy in Rheumatic Diseases. Edra, Milano 2010

3. Grassi W, Del Medico P. Atlas of Capillaroscopy. Edra, Milano 2004

4. Grassi W, Angelis R. Capillaroscopy: questions and answers. Clin Rheumatol 2007; 26: 2009-16.

5. Neubauer-Geryk J, Kozera GM, Wolnik B, et al. Decreased reactivity of skin microcirculation in response to L-arginine in later-onset type 1 diabetes. Diabetes Care 2013; 36: 950-6.

6. Pazos-Moura CC, Moura EG, Bouskela E, et al. Nailfold capillaroscopy in diabetes mellitus: morphological abnormalities and relationship with microangiopathy. Braz J Med Biol Res 1987; 20: 777-80.

7. Rossi M, Carpi A. Skin microcirculation in peripheral arterial obliterative disease. Biomed Pharmacother 2004; 58: 427-31.

8. Antonios TF, Singer DR, Markandu ND, et al. Structural skin capillary rarefaction in essential hypertension. Hypertension 1999; 33: 998-1001.

9. Humbert P, Sainthillier JM, Mac-Mary S, et al. Capillaroscopy and videocapillaroscopy assessment of skin microcirculation: dermatologic and cosmetic approaches. Cosmet Dermatol 2005; 4: 153-62. 
10. Gallucci F, Russo R, Buono R, et al. Indications and results of videocapillaroscopy in clinical practice. Adv Med Sci 2008; 53: 149-57.

11. Rajan V, Varghese B, van Leeuwen TG, Steenbergen W. Review of methodological developments in laser doppler flowmetry. Lasers Med Sci 2009; 24: 269-83.

12. Stücker M, Steinberg J, Memmel U, et al. Differences in the two-dimensionally measured laser Doppler flow at different skin localisations. Skin Pharmacol Appl Skin Physiol 2001; 14: 44-51.

13. Mrowietz C, Franke RP, Pindur G, et al. Reference range and variability of laser-Doppler-fluxmetry. Clin Hemorheol Microcirc 2017; 67: 347-53.

14. Cracowski JL, Minson CT, Salvat-Melis M, Halliwill JR. Methodological issues in the assessment of skin microvascular endothelial function in humans. Trends Pharmacol Sci 2006; 27: 503-8.

15. Roustit M, Blaise S, Millet C, Cracowski JL. Reproducibility and methodological issues of skin post-occlusive and thermal hyperemia assessed by single-point laser Doppler flowmetry. Microvasc Research 2010; 79: 102-8.

16. Blaise S, Hellmann M, Roustit M, et al. Oral sildenafil increases sodium nitroprusside iontophoresis induced skin hyperaemia in healthy volunteers. Br J Pharmacol 2010; 160: 1128-34.

17. Ljung $\mathrm{P}$, Bornmyr S, Svensson $\mathrm{H}$. Wound healing after total elbow replacement in rheumatoid arthritis. Acta Orthop Scand 1995; 66: 59-63.

18. Rosato E, Borghese F, Pisarri S, Salsano F. Laser Doppler perfusion imaging is useful in the study of Raynaud's phenomenon and improves the capillaroscopic diagnosis. J Rheumatol 2009; 36: 2257-63.

19. Lahiri BB, Bagavathiappan S, Jayakumar T, Philip J. Medical applications of infrared thermography: a review. Infrared Phys Technol 2012; 55: 221-35.

20. Sivanandam S, Anburajan M, Venkatraman B, et al. Medical thermography: a diagnostic approach for type 2 diabetes based on non-contact infrared thermal imaging. Endocrine 2012; 42: 343-51.

21. Balbinot LF, Canani LH, Robinson CC, et al. Plantar thermography is useful in the early diagnosis of diabetic neuropathy. Clinics 2012; 67: 1419-25.

22. John HE, Niumsawatt V, Rozen WM, Whitaker IS. Clinical applications of dynamic infrared thermography in plastic surgery: a systematic review. Gland Surg 2016; 5: 122-32.

23. Fife CE, Smart DR, Sheffield PJ, et al. Transcutaneous oximetry in clinical practice: consensus statements from an expert panel based on evidence. Undersea Hyperb Med 2009; 36: 43-53.

24. Ladurner R, Küper M, Königsrainer I, et al. Predictive value of routine transcutaneous tissue oxygen tension (tcp02) measurement for the risk of non-healing an amputation in diabetic foot ulcer patients with non-palpable pedal pulses. Med Sci Monitor 2010; 16: 273-7.

25. Moosa HH, Peitzman AB, Makaroun MS, et al. Transcutaneous oxygen measurements in lower extremity ischemia: effects of position, oxygen inhalation, and arterial reconstruction. Surgery 1988; 103: 193-8.

26. Fife CE, Buyukcakir C, Otto GH, et al. The predictive value of transcutaneous oxygen tension measurement in diabetic lower extremity ulcers treated with hyperbaric oxygen therapy: a retrospective analysis of 1144 patients. Wound Rep Reg 2002; 10: 198-207.
27. Papa G, Spazzapan L, Pangos M, et al. Compared to coverage by STSG grafts only reconstruction by the dermal substitute Integra ${ }^{\circledR}$ plus STSG increases TcPO2 values in diabetic feet at 3 and 6 months after reconstruction. G Chir 2014; 35: 141-5. 